\title{
STRUCTURE AND COMPOSITION OF WHITE DWARF ATMOSPHERES AND CONVECTION ZONES
}

\author{
K.H. BOHMM \\ Astronomy Department \\ University of Washington
}

\begin{abstract}
Summary
We present a brief review of the basic properties of white dwarf atmospheres, convection zones and corona models emphasizing qualitative and intuitive aspects.

1. Atmospheres: We restrict our discussion essentlally to very hot and very cool atmospheres since these are especially interesting. With regard to the first type of objects we study the fundamental differences between $D A$ and non-DA models and between their surface fluxes. We discuss the important role of electron scattering in determining the EUV spectra of these objects. The differences between DAs and non-DAs with regard to backwarming effect and surface cooling are summarized.
\end{abstract}

In our discussion of very cool non-DA atmospheres we emphasize the importance of the additional energy transport mechanisms convection and conduction which should both be very effective for $\mathrm{T}_{\text {eff }}<4000 \mathrm{~K}$ and which lead to a very flat temperature gradient. This small gradient must lead to a rather featureless surface flux.

2. Convection zones. After a survey of the basic numerical results in this field we investigate the question whether convection In white dwarfs has the same basic properties as convection in other stars. We find that contrary to Intultive expectations the Rayleigh number in very cool non-DAs is higher than in the sun (indicating very turbulent convection). The Prandtl number in these objects is 6 to 7 orders of magnitude higher than in the sun.

3. Coronae. The basic methods of the calculation of coronae for white dwarfs are very briefly discussed. We present some results for DA and non-DA stars from unpublished work by D.O. Muchmore and the author. It uses revised values for the emissivities. Only 
non-DA coronae are of practical interest. DA coronae have much lower densities and temperatures. White dwarf coronae do not generate a stellar wind.

\section{Introduction}

In this paper we intend to present a broad and essentially qualitative or semi-qualitative overview of the structure of white dwarf atmospheres and envelopes as a function of $T_{e f f}$ and chemical composirion. We shall try to emphasize the intuitive explanation and understanding of effects predicted by model atmosphere and convection zone calculations.

I feel strongly that such a qualitative understanding is important and necessary and that it is less developed for envelopes and atmospheres of white dwarfs than for other stars. This is probably due to the fact that at the time when serious discussions of white dwarf atmospheres were inftiated fairly sophisticated model atmosphere computer programs were already available. This led to attempts to explain observed details for individual stars almost immediately and it sometimes seems that we never found the time to develop the basic understanding of white dwarf atmospheres with the same depth as it is undoubtedly avallable in the case of many other types of stellar atmospheres. This may be espectally dangerous since we may overlook the possibility of unexpected physical effects resulting from the high surface gravity and/or the unusual chemical composition of white dwarfs. In order to indicate the type of question which we should study let me simply state two completely arbitrary examples:

1. Does convection in, say, cool white dwarfs with their extremely small scale heights ( $10 \mathrm{~m}$ ) really have the same basic properties (like extremely large Rayleigh number and extremely small Prandt number) which are typical for the usual stellar convection zones?

2. What is the physical explanation of the secondary maximum of the surface flux $F_{y}(0)$ at EUV or soft $X$-ray frequencies which occurs in certain hot white dwarf models but not in others? Which selection of parameters makes this secondary peak especlally strong?

It is obvlous that an answer to questions of this type would not only be of didactic value but could also have considerable practical Importance.

The plan for my presentation is as follows. In section II I shall present a very brief summary of our knowledge of the chemlcal composition of white dwarf atmospheres. I can be very brlef here since we have heard quite a number of talks at this conference in which this topic has been addressed. In sections III and IV I shall discuss the atmospheres and convection zones of white dwarfs. In view of our own interests I shall add to this a chapter $V$ in which we shall consider briefly certain semi-quantitative estimates of the properties of predicted coronae for non-DA and DA white dwarfs. This 
chapter will certainly be more "academic" and also more controversial than the other ones. However, these results are also interesting and merit further discussion.

\section{Chemical Composition}

The distinction between DA (consisting of almost pure H) and nonDA white dwarfs (consisting of almost pure He) is well known. Very low upper limits for the $H$ abundance in non-DAs and for the He abundance in hot DA's have been set and are typlcally of the order of $10^{-4}$ or lower. Llebert (1977) has been able to determine a hydrogen abundance of $3 \times 10^{-4}$ in the non-DA star R640. Elements like Ca, Fe, $\mathrm{Mg}, \mathrm{Na}$ seem to show abundances between $10^{-3}$ and $10^{-4}$ of the solar value e.g.In van Maanen 2, R640, L745-46H (see Wegner 1972, Grenfell 1974), but there is also the case of G165-7 (Wehrse and Llebert 1979) In which these abundances are about $3 \times 10^{-2}$ of the solar value. $\mathrm{C}, \mathrm{N}, 0$ abundances are usually not well determined and only some not very restrlctive upper limits can be given. Upper limits of the order of $1 \times 10^{-4}$ (corresponding to $\sim 0.1$ of the solar value) are typical and are often determined indirectly from the number of free electrons in the atmosphere (cf. Wegner 1972, Grenfell 1974). However, it has also been stated that $C, N, O$ are strongly underabundant (Fontaine and Michaud 1979). It has been remarked repeatedly that the depletion factors for the individual metals are not very different from each other.

Some aspects of these results are 1 llustrated in $\mathrm{flg}$. I where we show the abundances (and upper limits of abundances) in some selected non-DA stars, namely van Maanen 2 (Weldemann 1960, Wegner 1972, Grenfell 1974), Ross 640 (Llebert 1977), G165-7 (Wehrse and Liebert 1979) and the $\lambda 4670$ star G47-18 (Bues 1973) and compare them to solar abundances. The diagram shows the apparent simflarity of the relative distribution of the elements $\mathrm{Na}$ to Fe in different non-DA's (which have rather different absolute metal abundances) and the similarity of all these distributions to the relative metal abundances in the sun. This fact somewhat simplifies the discussion of the influence of the abundances in white dwarf envelopes.

It is interesting to note that recently Koester, Liebert and Hege (1979) have found definite evidence of $\mathrm{H} / \mathrm{He}$ abundance ratios in DO stars wh1ch are intermedlate between DA and non-DA abundances. In $\mathrm{HZ} 21\left(\mathrm{~T}_{\text {eff }} \sim 4.8 \times 10^{4} \mathrm{~K}\right.$ ) they find $\mathrm{N}_{\mathrm{He}} / \mathrm{N}_{\mathrm{H}} \sim 6$, in $\mathrm{HZ} .34$ ( $\mathrm{T}_{\text {ef }}$ $\sim 5 . \times 10^{4} \mathrm{~K}$ ) the result is $2 \times 10^{-2}$. Though the error bars stil correspond to approximately one power of ten in each direction, the result is highly interesting since (especially in the case of Hz21) it excludes a typical DA as well as a non-DA composition. 


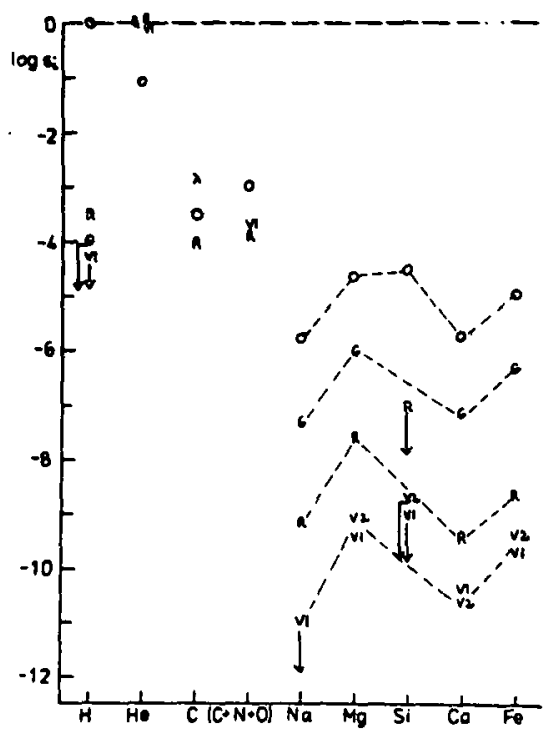

Fig. 1. Element abundances in a number on non-DA stars compared to solar abundances (open circles). The symbols have the following meaning: $R=$ Ross 640 (Liebert 1977), $G=$ Gl65-7 (Wehrse and Liebert 1979), $\lambda=$ G47-18 (Bues 1973), V1, V2 = van Maanen 2 (Wegner 1972, Grenfell 1974).

An intriguing problem is presented by the $\lambda 4760$ stars, non-DA stars with a somewhat enhanced carbon abundance (Bues 1973, Grenfell 1974) which also have a $\mathrm{C} / 0$ ratio of at least 12 (Bues 1973). Though the carbon abundance in these objects differs only slightly (If at a11) from the solar value ( $f \mathrm{~g}_{\mathrm{g}}$. I) the fact that we have an additional electron source (In comparison to other white dwarfs in the same $T_{e f f}-$ range) is of great interest and may lead (depending on the exact value of the $C$ abundance) to considerable changes in the atmosphere and convection zone structure (Muchmore and $B 8 h m$ 1977) which may even have some noticable influence on the cooling times of these objects.

This very brief and somewhat superficlal review of the white dwarf abundance problem should suffice as a starting point for the following discussion of atmospheres convection zones and coronae of white dwarfs.

\section{White Dwarf Atmosphere Structure}

General Remarks. Our discussion will be based on the assumption that the most interesting problems presently occur in connection with

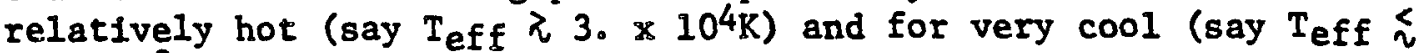
6 . $x 10^{3} \mathrm{~K}$ ) white dwarfs. In view of the space limitation for this article and in view of our own interest it may be useful to adopt this point of view though the philosophy behind it may be controversial. Our approach should in no way distract from the great 
practical 1mportance of the studies of white dwarfs in the middle range of $\mathrm{T}_{\text {eff }}$. We shall only mention the classical work by Weidemann (1963), Terashita and Matsushima (1969), Bues (1970), Strittmatter and Wickramasinghe 1970 and the modern work by Grenfell (1974), Wehrse (1971, 1974, 1976a,b), Koester, Schulz and Weldemann (1979). The special properties of white dwarf atmospheres and convection zones are due to thelr high surface gravity and their unusual chemical composition. Though these two effects cannot be clearly separated it is well known that drastic high density effects are essentially restricted to very cool white dwarfs and among these especially to the cool non-DAs. The typical properties of hot white dwarfs are (within certain limits) strongly determined by their pecultar chemical composition. It is well-known that in this context the lack of continuous absorption at small wavelengths in hot DA stars leads to a number of interesting effects which do not occur in other stars. We shall now first discuss the atmospheres of very hot white dwarfs (with, say, 3. $x 10^{4} \leqslant \mathrm{~T}_{\text {eff }}$ $\{7 . x 104 \mathrm{~K}$ ) and then consider briefly the intriguing problems connected with very cool white dwarf atmospheres.

Atmospheres of Hot White Dwarfs. Model atmospheres for hot white dwarfs have recently been calculated by a large number of authors including Auer and Shipman (1977), Shipman (1976), Koester, Liebert and Hege (1979), Wesemael (1978, 1979) Weidemann and Koester (1979), $B$ bhm and Kapranidis (1979). One of the more Impressive aspects of these results is the great difference of the model atmospheres and surface fluxes $F_{v}(0)$ for $D A s$ and for non-DAs indicating the importance of the chemical composition. In order to separate surface gravity and composition effects B Bhm and Kapranidis (1979) have calculated model atmospheres and $F_{\gamma}(0)$ for white dwarfs with DA, non-DA, some intermediate as well as solar abundances always using the same method. Though our method is based on Lucy's temperature correction procedure and does not usually achieve the same degree of accuracy as calculations based on the complete linearization combined with the varlable Eddington factor method (Auer and Mihalas 1969, 1970) we shall base the following qualitative discussion on these models. Our main argument is that for a qualitative understanding of the basic effects a very high accuracy in the radiative transfer (and radiative equilibrium) equations is not required but that a large set of models with different compositions is very important for the present discussion. These non-gray L.T.E. models have been iterated to a flux constancy of 0.5 to $1 \%$ in the interval $0 \leq \bar{\tau} \leq 10.0$. $A$ weak convection zone in these layers appears only in non-DA models with $\mathrm{T}_{\text {eff }}<5.104 \mathrm{~K}$. We find fundamental differences between $\mathrm{DA}$, non-DA models and models with normal composition. An essential part of these differences is attributable to the fact that hot DA atmospheres are very "peculiar" in the sense that their absorption coefficient for $\lambda \ll 912 \AA$ is very small leading to a great importance of absorption edges even of trace elements and of electron scattering effects in the EUV. It is very interesting to note that electron scattering is inportant (In this sense) for white dwarfs though we normally assume that electron scattering is typical for low density situations. Electron scattering leads (at least in our own models) to the intermediate maxima in the surface flux which 

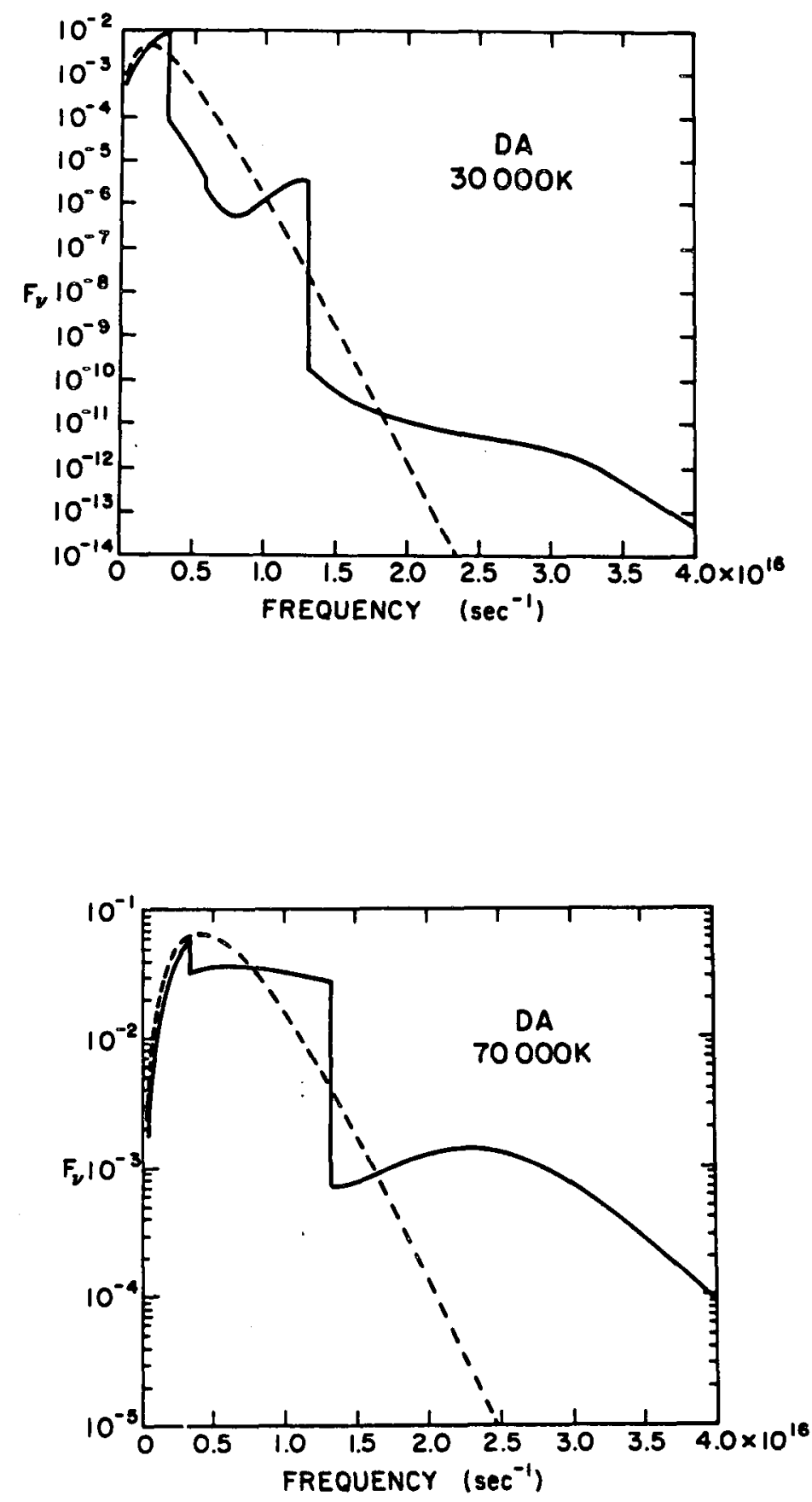

Fig. 2. Frequency dependence of the surface flux in hot DA stars. The broken line corresponds to the black body radiation for $T=T_{\text {eff }}$. (From BHhm and Kapranidis 1979). 

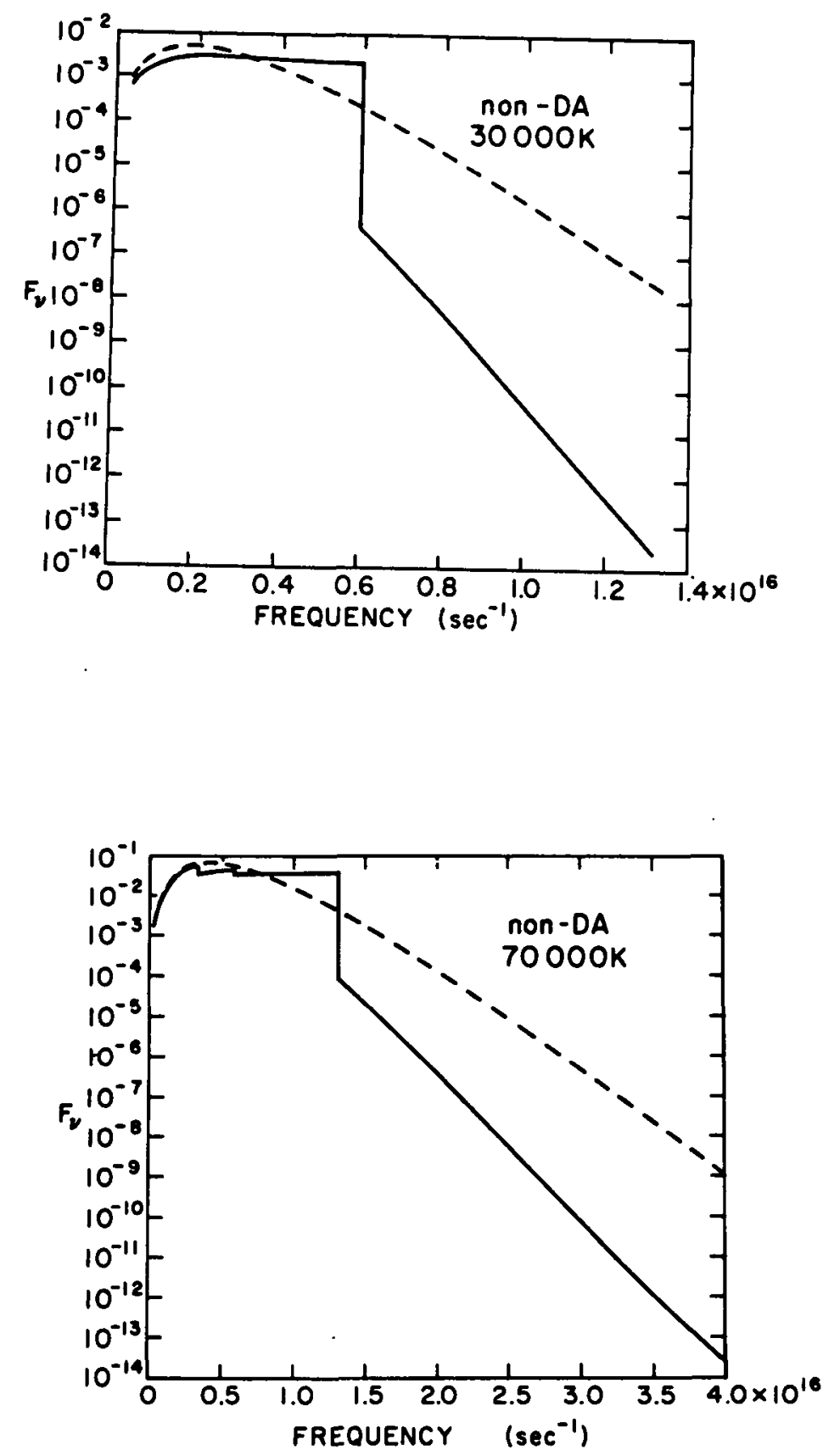

Fig. 3. Surface flux for hot non-DA stars. Analogous to fig. 2. (Bלhm and Kaprantdis 1979). 

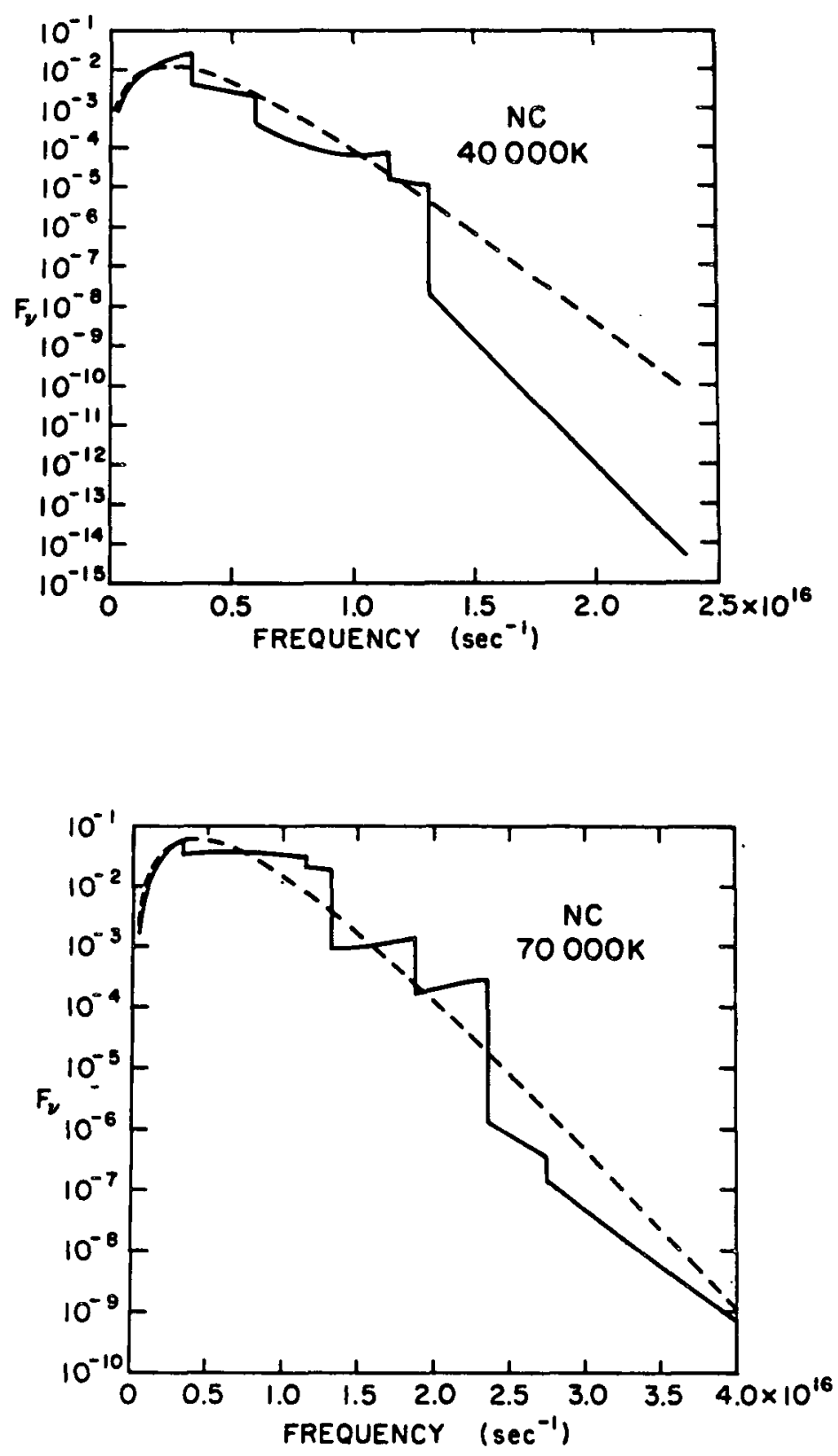

F1g. 4. Surface flux for (fictitious) hot white dwarfs with solar composition (see text). Analogous to $\mathrm{fig}$. 2, but note the different effective temperature for the upper part of the dlagram. 
have played such an important role in the photospheric interpretation of the EUV radiation from hot white dwarfs like Sirius $B$ and $\mathrm{HZ} 43$ (cf. Shlpman 1976, Cash et al. 1978). From our calculations we deduce that the enhanced radiation in the EUV range which occurs in a wide range of $T_{\text {eff }}$ (see fig. 2) Is due to the following effect: In the ultraviolet part of the spectrum (1.e. for wavelengths which are far. shorter than the one corresponding to the maximum of the Planck function) $B_{V}$ becomes an extremely steep function of the optical depth. This has the consequence that in the upper layers of the atmosphere the mean Inensity $\bar{I}_{v}\left(\tau_{v}\right)$ becomes very much larger than $B_{v}\left(\tau_{v}\right)$. This effect increases with increasing $v$ and reaches several orders of magnitude. As the ratio of the scattering coefficient $\sigma_{v}$ to the absorption coefficient $K$ increases with increasing $\nu$ the source function $S$ is more and more influenced by $\bar{I}_{\nu}$ leading to an increase in $S_{V}$ with ${ }^{\nu}$. This increase is then reflected in an increase of $F_{1}(0)$ with $v$ leading to the secondary maxima shown in $\mathrm{fig} .2$. As shown in fig. 3 (referring to non-DA stars) and in fig. 4 ("normal composition" stars) there is no such effect in non-DA and normal composition stars. In these stars electron scattering is unimportant and the EUV radiation always remains below the corresponding blackbody radiation, an effect well-known from the study of central stars of planetary nebulae. One of the curiosities resulting from the facts discussed above and from the relatively low density in hot DA atmosperes is the fact that the HeII Lyman edge is more pronounced in a DA star of $30000 \mathrm{~K}$ than in the corresponding non-DA star provided the He abundance in the DA star is at least $10^{-4}$. The very different behavior of the atmospheric densities at $\bar{\tau}=0.1$ and $\bar{\tau}=1.0$ as a function of $T_{\text {eff }}$ for $D A$ and non-DA stars is illustrated in fig. 5 . The density increase with $T_{\text {eff }}$ for DA stars is obviously a consequence of the decline of the opacity $\bar{k}$ with increasing temperature. The deep minimum in the $\bar{\tau}=1.0$ density curves for non-DAs between, say, $T_{\text {eff }}=$ 4. $x 10^{4} \mathrm{~K}$ and $\mathrm{T}_{\text {eff }}=7 . \times 10^{4} \mathrm{~K}$ is obviously a consequence of the very strong HeII absorption in this range.

In order to obtain an overview over the basic properties of hot white dwarf atmospheres without a real listing of model atmospheres we have drawn diagrams of the backwarming and surface cooling effects in DA, non-DA and normal composition stars as a function_of $T$ eff. We have simply done this by plotting $T_{1} / T_{\text {eff }}$ (with $T_{1}=T(\tau=1)$ and $\mathrm{T}_{\rho} / \mathrm{T}_{\text {eff }}$ (with $\mathrm{T}_{0}=$ surface temperature) as a function of $\mathrm{T}_{\text {eff }}$. It should be noted that these effects are due to the extreme non-grayness of the continum only since line blanketing has not been taken into account. As an example of the types of results which are obtained we show the backwarming effects for all three compositions in fig. 6. We find a very strong similarity between DA white dwarfs and high gravity stars with "normal" (solar) composition. This indicates that the backwarming is essentially controlled by the hydrogen absorption edges in both cases though between $4 \times 10^{4} \mathrm{~K}$ and $6 \times 10^{4} \mathrm{~K}$ there is a slight lowering of the backwarming in DA stars due to the essential absence of absorption edges of NIII, CIII, OIV, NIV and the reduced importance of the HeII Lyman edge. 

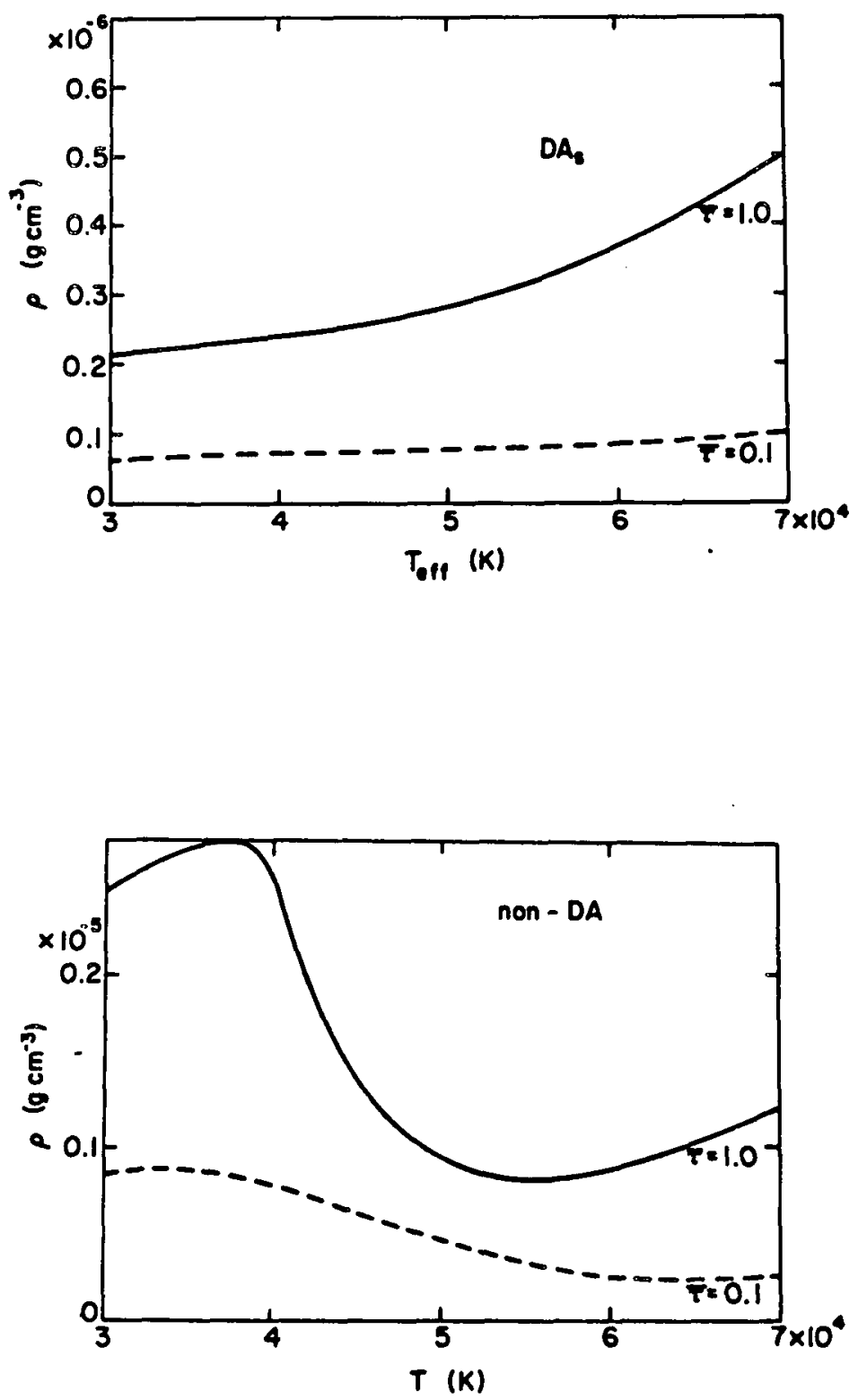

Fig. 5. Atmospheric densities as a function of $T_{\text {eff }}$ for $D A^{\prime} s$ and non-DA's. (Bלhm and Kapranid1s 1979). 


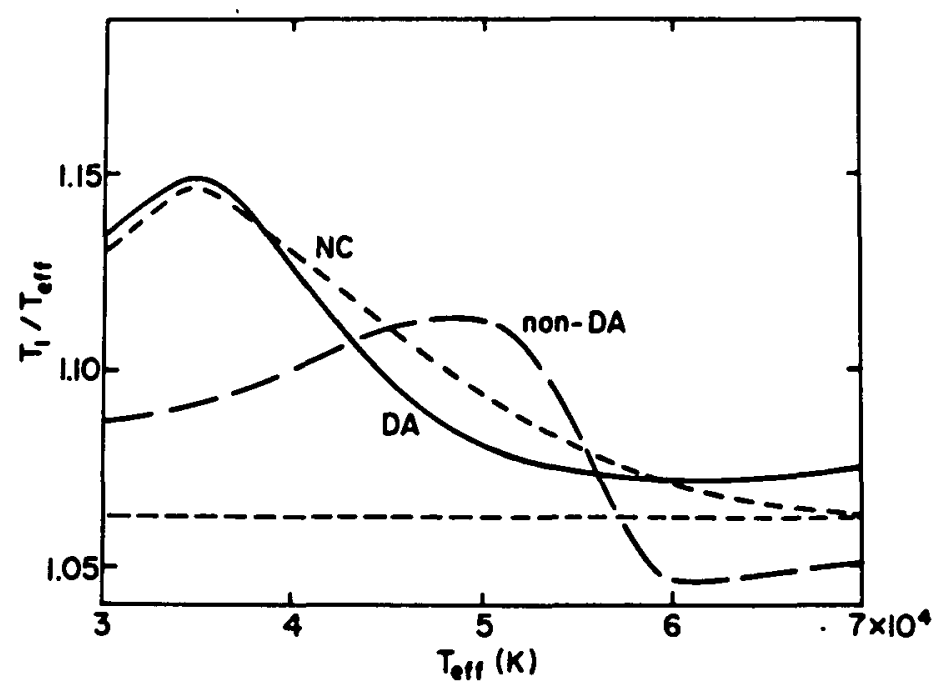

Fig. 6. A comparison of the backwarming effect (due to the nongray continuum only) for DA's, non-DA's and for fictitlous white dwarfs with solar composition (NC). See text.

The backwarming effect in hot non-DAs looks very different. In the range $3 \times 10^{4} \lesssim \mathrm{T}_{\text {eff }} \leqslant 4 \times 10^{4} \mathrm{~K}$ it is considerably smaller than in DA stars because of the fact that all important absorption edges occur essentially outside the frequency range which contains the largest part of the radiative flux. Around $50000 \mathrm{~K}$ the backwarming becomes large because the maximum of the flux is now shifted into the frequency region of the HeI Lyman edge and consequently the atmosphere becomes highly non-gray. Eventually for $\mathrm{T}_{\text {eff }} \approx 6 \times 10^{4} \mathrm{~K}$ the backwarming becomes "negative" (1.e. $T_{1} / T_{\text {eff }}$ is smaller than for a gray atmosphere). This type of basic effect has been discussed by Unno (1963, see also Bbhm and Deinzer 1966). This occurs if a considerable part of the "nongrayness" occurs in the "red" part of the spectrum (1.e. at wavelengths which are longer than that for the maximum of the flux) and can be understood in a purely qualitative way. It is interesting to note that this effect does not occur in hot DA stars. A number of other important properties of hot white dwarfs have been discussed in the same qualitative way by B $8 \mathrm{hm}$ and Kapranidis (1979).

Atmospheres of Very Cool non-DA White Dwarfs. In view of the great importance of understanding the final cooling phases of white dwarfs a study of the atmospheres of very cool white dwarfs is of great interest. There are a number of reasons for starting such an investigation by looking first at non-DA stars:

1. There is a very good chance that because of convective mixing at fairly low $T_{\text {eff }}$ (Koester 1976) the fraction of non-DA's among the cool white dwarfs will be considerably higher than for hot degenerates. 
2. From a theoretical point of view the study of cool non-DAs is less complex since there is no complex chemistry involved.

3. The interesting physical effects connected with extremely high pressure in the atmosphere occur already at a higher $T_{\text {eff }}$ in non-DAs than in DAs.

Atmospheres, convection zones and internal temperatures of these very cool ( $\left.\mathrm{T}_{\text {eff }}<6000 \mathrm{~K}\right)$ non-DA stars have first been studied by B Bhm, Carson, Fontaine and Van Horn (1977). It was found (see also B Bhm, 1976) that such atmopsheres are almost completely convective. (Convection starts at $\bar{\tau} \sim 0.01$ and higher). The convection is extremely effective. At $\bar{\tau} \sim 1.0$ approximately $60 \%$ of the energy is transported by convection. Obviously this value is very much higher than in any other stellar atmosphere and is also higher than anything considered possible some time ago. Densities in these atmospheres reach values of the order of $1 \mathrm{~g} \mathrm{~cm}^{-3}$ near $4000 \mathrm{~K}$. Below $\mathrm{T}_{\text {eff }} \sim 3800 \mathrm{~K}$ pressure ionization and partial degeneracy becomes important in the atmosphere and the physics becomes very different from that of normal stellar atmospheres. The calculation of the equation of state, the opacities, the energy transport, line broadening and line formation should in principle be completely reconsidered. At the present time this has not yet been done. However, we consider this as one of the important white dwarf atmosphere projects for the future. Even in order to find out whether white dwarfs with $\mathrm{T}_{\text {eff }} \lesssim 4000 \mathrm{~K}$ exist (see Liebert et al. 1979 for an observational discussion of this problem) or not it will be necessary to know something about their atmosphere theory. Only with this information shall we know what to look for.

A first small step in this direction is being done by Blhm, and Muchmore (unpublished) by studying the energy transport and temperature stratification in very cool non-DA atmospheres. Using an equation of state and opacity calculations based on the hot Thomasfermi model calculations by Carson (unpublished) as in B bhm et al. (1977) we are trying to construct model atmospheres taking into account radiative, convective and conductive energy transport due to partially degenerate electrons simultaneously. Some very preliminary results for an extreme atmosphere of $T_{\text {eff }}=3000 \mathrm{~K}$ are shown in $\mathrm{fig}$. 7. The results are still very unsatisfactory since the total flux is constant only to within somewhat more than $10 \%$. Nevertheless it may be useful to consider these results as a qualitative description of the structure of such a very cool atmosphere. In this model the convective flux rises monotonically, starting almost at the surface. The gradient of the convective flux increases rapidly near $\tau \sim 1.0$ as a consequence of the decreased radiative exchange in the optically thick layers. The conductive flux first increases rapidly as a function of $\bar{\tau}$ because of the rapidly increasing density, the resulting increasing pressure ionization and the resulting increasing degeneracy. Below $\bar{\tau} \sim 0.7$ the conductive flux $F_{\text {fond }}$ decreases again though the conduct1vity continues to increase. IhIs is due to the rapid flattening of the temperature gradient as a consequence of the rapid increase in the effectiveness of convection. In the right hand part of fig. 7 we compare the temperature stratification for 1 . pure radiative 
equilibrium, 2. radiative plus convective energy transport, and 3. radiative + convective + conductive energy transport. The transition from 1. to 2. to 3 . leads of course to an increased flattening of the temperature stratification. In the case 3 . the temperature stratification is so flat that we have an almost isothermal atmosphere, meaning that if we have L.T.E. (which must be true at these very high densities) the surface flux can devlate only slightly from black-body radiation and lines should be extremely shallow even if we would ignore the extreme pressure broadening effects. Note that the total variation of the temperature between $\bar{\tau}=0$ and $\bar{\tau}=1.8$ is only of the order of $200 \mathrm{~K}$.

Convection Zones. It is well known that the basic possibility of convection in white dwarfs was first considered by Schatzman (1958). The relevance of the convection zone to the cooling problem was first considered and detailed numerical models of convection zones were first calculated by Bbhm (1968, 1970), Greenstein (1969), Van Horn (1970), $\mathrm{B}$ bhm and Cassinelli (1971a), B bhm and Grenfell (1972) and Koester (1972). The great importance of white dwarf convection zones in connection with the mixing of elements was first emphasized by Strittmatter and Wickramasinghe (1971). The acoustic noise generation in white dwarf convection zones and its relevance to the formation of coronae in non-DA stars has first been discussed by Blhm and Cassinelli $(1971 a, b)$. The most detailed and complete study of chemically homogeneous convection zones in DA, non-DA stars and in almost pure carbon layers was carried out by Fontaine (1973) and Fontaine and Van Horn (1976), (see also Fontalne et al. 1974). These papers gave us an excellent overview over the presence of convection zones in different types of white dwarfs and have continued to supply us with important data about these convection zones. We have learned from this and more recent work that atmospheric convection vanishes for $T_{\text {eff }} \sim 16500 \mathrm{~K}$ in $D A$ and for $T_{\text {eff }} \sim 50000 \mathrm{~K}$ in non-DA stars. It was also found that convection zones in carbon layers are thinner than expected since only a transition through low ionization stages leads to a convective instability. As soon as a sizeable density of free electrons has been bullt up the strong heat conduction by degenerate electrons lowers the temperature gradient so much that the convective instability disappears.

The calculations of convection zones were put on a new basis by the work of Koester (1976) who calculated convection zones using a Henyey code and taking into account the original hydrogen-helium stratification and convective mixing effects. Related calculations have been carrled out by d'Antona and Mazzitell1 (1975, see also 1979). This work shows the possible strong suppression of convection by the presence of rapid variations of the molecular weight and opacity due to the transition from the He-rich to the H-rich zone. The most important result is that the possibility of the conversion of a DA to a non-DA star occurs only well below $\mathrm{T}_{\text {eff }} \sim 10^{4} \mathrm{~K}$ for practically all reasonable thicknesses. Similar calculations for stratified carbonhellum envelopes have been carried out by d'Antona and Mazzitelli (1979) and by Vauclair and Fontaine (1979) in connection with the question whether the existence of $\lambda 4670$ stars can be explained by 

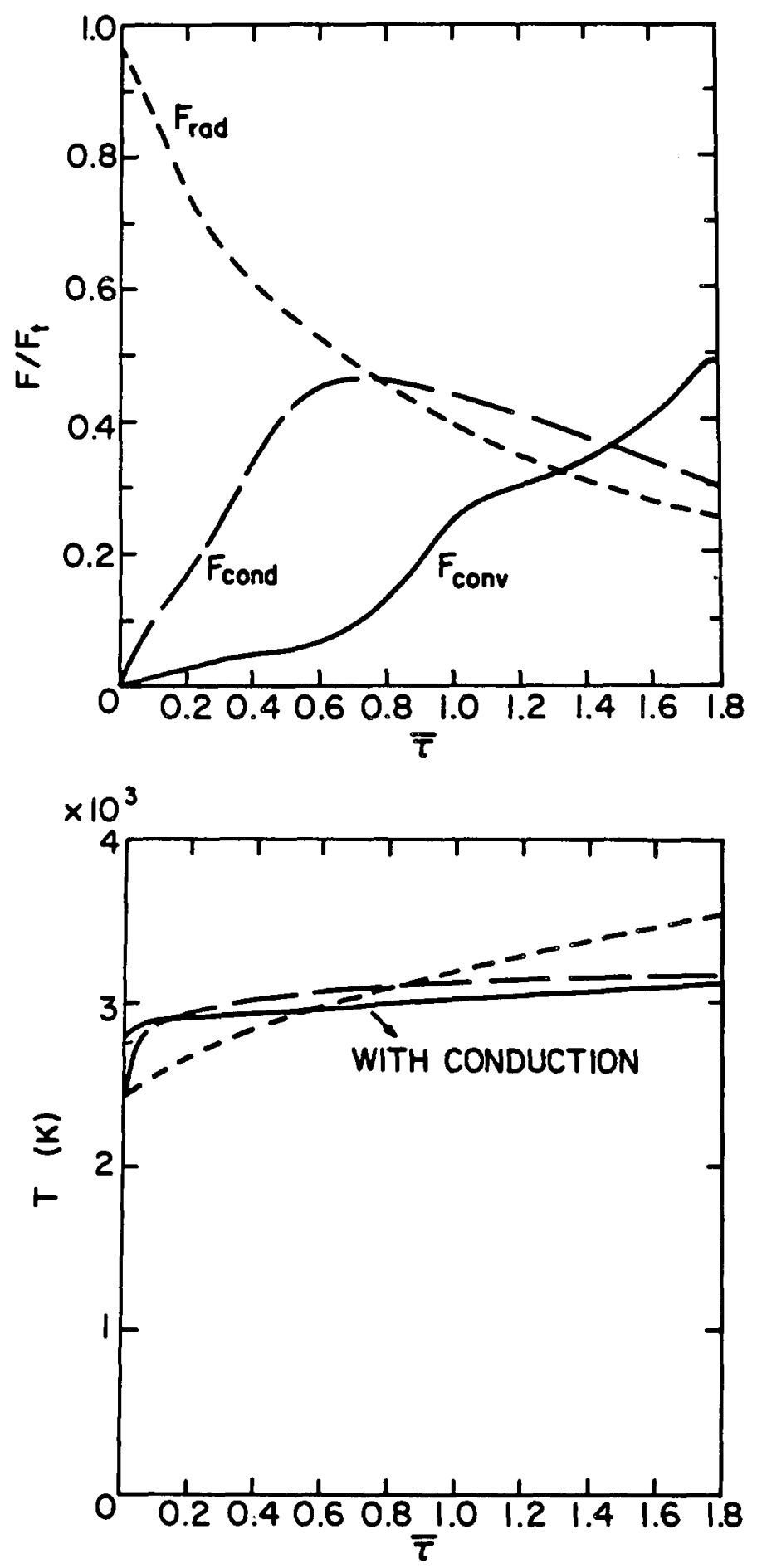

Iig. 7. Energy transport as a function of optical depth (upper part of diagram) and temperature stratification (lower part) for a very $\operatorname{cool}\left(\mathrm{T}_{\text {eff }}=3000^{\circ} \mathrm{K}\right.$ ) pure He white dwarf atmosphere (for $g=8$ )。 In the lower part the short broken line corresponds to a purely radiative model, long dashes show the model including convection and the solid Iine gives the model which takes into account radiation plus convection plus conduction. The model, calculated by Muchmore and Bbhm, has to be considered as tentative. 
convective mixing. From the point of view of our present knowledge of white dwarf convection, papers like these seem to present the final answers to the convective mixing problem in white dwarfs. However, some caution may not be superfluous. We have to admit that all these calculations are based on the very simplest form of the convection theory and the possible convection instabilities in "doublediffusive" convection layers are rather complex (cf. Huppert 1977). Moreover, it has not yet been fully investigated which role convective overshoot (cf. Bbhm 1963, Shaviv and Salpeter 1973) may play in these processes. This last point is of course important in view of the fact that very slow convection may already lead to considerable mixing.

As a last point of this I would like to discuss the question (mentioned earlier) whether convection in white dwarfs really has, in spite of the small geometrical scale and the very high density (especially in cool white dwarfs), the same basic physical properties as convection in, say, main sequence stars. Some people have the feeling that convection, say, in a star like van Maanen 2, may be almost laminar because of the very small scales. It will be instructive in this connection to look at the Rayleigh number and the Prandtl number in the upper part of white dwarf convection zones. As you remember the Rayleigh number ( $\mathrm{Ra}$ ) is essentially the ratio of the buoyancy to the viscous forces. It has to be of the order of $10^{6}$ for the convection to become really turbulent. In the upper parts of the solar convection zone ( $\mathrm{Ra}$ ) $\sim 10^{14}$. The Prandtl number $\sigma$ is the ratio of the radiative plus thermal conductivity and the kinematic viscosity and is typically very small in stellar atmospheres leading to convective properties which are very different from those in laboratory situations. Our question is: Does convection in white dwarfs have similar Rayleigh and Prandtl numbers as that in other stars? We have calculated these characteristic numbers for non-DA star convection zones in the range $3000 \mathrm{~K} \leq \mathrm{T}_{\text {eff }} \leq 30000 \mathrm{~K}$ using models by $\mathrm{B}$ Hhm and Cassinelli (1971) in the range $6500 \mathrm{~K} \leq \mathrm{T}_{\text {eff }} \leq 30000 \mathrm{~K}$ and by $\mathrm{B} 8 \mathrm{hm}$ et al. (1977) in the very low temperature range $3000 \mathrm{~K} \leq \mathrm{T}_{\text {eff }} \leq 6000 \mathrm{~K}$. The results are shown in $\mathrm{fig}$. 8 .

Though the physical assumptions used in the calculation of the equation of state and the opacities are rather different in both series of models there seems to be an almost continuous transition between the characteristic numbers of the two sets of models. Since white dwarf convection zones cannot be described by the Boussinesq approximation we have to specify for which layer the numbers ( $R a$ ) and $\sigma$ have been calculated. We have always selected the point at which the convective velocity $v$ has reached its maximum $v_{\max }$. The results presented in fig. 8 are surprising at first sight. They show that the Rayleigh numbers are generally large and increase very rapidly towards smaller $T_{e f f}$. In fact, for very cool white dwarfs the Rayleigh number is about ten powers of ten higher than in the solar convection zone. It is intriguing that in this sense very cool white dwarfs (in spite of their very small scale heights and their small convection zone thickness) have more fully developed turbulence in their convection zones than the sun. The main (though not the exclusive) reason for this is the fact that the radiative (plus thermal) conductivity is 

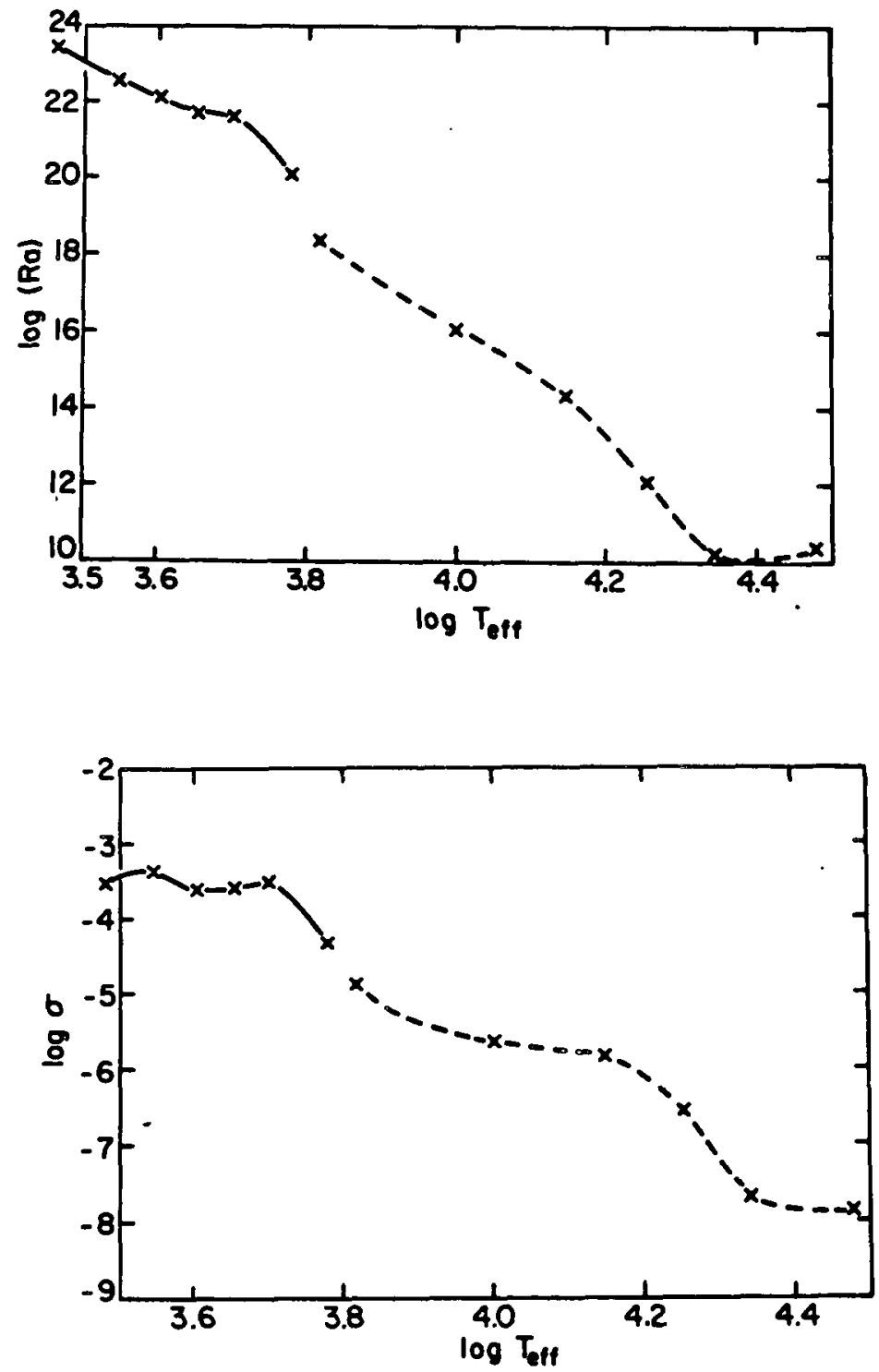

Fig. 8. The Rayleigh number (Ra) and the Prandtl number ( $\sigma$ ) for non-DA convection zones as a function of $\mathrm{T}_{\text {eff }}$. The numbers have been calculated for the layer in which the convective velocity reaches its maximum. Solid curves are based on convection zone models by B bhm et al. (1977), broken curves on those by Bbhm and Cassinelli (1971). 
reduced by more than ten powers of ten in comparison to the sun.

The Prandtl number for cool non-DA convection zones is much higher than for the sun and In this sense the convection is really slightly more similar to the laboratory case than in other astrophysical cases. Nevertheless $\sigma$ is still small and we still have a "typically astrophyslcal" situation. For high $T_{\text {eff }}$ the Prandtl number decreases rapid1y and is only one power of ten higher than in the sun for $T_{\text {eff }}$ ? 3. $x 104 \mathrm{~K}$.

\section{White Dwarf Coronae}

In this chapter we shall restrict ourselves to "classical corona formation". This means that the mechanical (acoustic) energy flux generated by the turbulence in the convection zone according to Lighthill's theory (cf. 1955) (see also Blermann and LUst 1960) is the main energy source for the corona formation. It is well-known from solar physics that the real situation 1s, at least, much more complicated. Nevertheless it is useful to consider the predictions from this type of theory for stars other than the sun. This should be done using the Kuperus-Ulmschneider approach (cf. Kuperus 1965, Ulmschneider 1967, De Loore 1970). A simpler but more controversial approach is the use of the minimum flux method (Hearn 1975). This method has been applied to a number of stars of different spectral type and luminosity by Hearn (1975) and by Mullan (1976). Muchmore and B Bhm (1978) have applied it to non-DA white dwarfs. Th1s requires some modifications in the method in order to take into account properly effects of the high gravity and the unusual chemical composition. Hearn's method has recently been criticized by Antiochos and Underwood (1978). Though their critlcism is basically justifled it is not yet clear whether results derived from Hearn's method are really not useable as crude qualitative approximations especially since the numerical results always look intuitively reasonable and since the acoustic fluxes which enter into the theory are very uncertain anyhow.

Very recently Muchmore and $B$ bhm have repeated the calculations for non-DA stars and also carried out analogous computations for DA stars using in both cases improved values of the emissivities. The results are presented in fig. 9 and 10, showing the coronal base pressure and the coronal temperature as a function of the effective temperature for non-DAs ( $f i g .9)$ and DA's ( $f 1 g, 10)$. The temperature of a non-DA corona at Teff $22.2 \times 10^{4}$ is now $4.5 \times 10^{6} \mathrm{~K}$ and at $\mathrm{T}_{\text {eff }} \sim 3 . \times 10^{4}$ we find a coronal temperature of $5.2 \times 10^{6} \mathrm{~K}$. As we might have expected, coronae of DA stars occur(if at all) only on a very narrow $T_{\text {eff }}$ range between 10000 and $11000 \mathrm{~K}$. The coronal temperature hardly reaches $4 \times 105 \mathrm{~K}$. These calculations show that while it should be basically possible to detect "classical" non-DA coronae in the effective temperature range $1.7 \times 10^{4} \leqslant \mathrm{~T}_{\text {eff }} \leqslant 3 \times 10^{4} \mathrm{DA}$ coronae seem to be of no practical interest. 

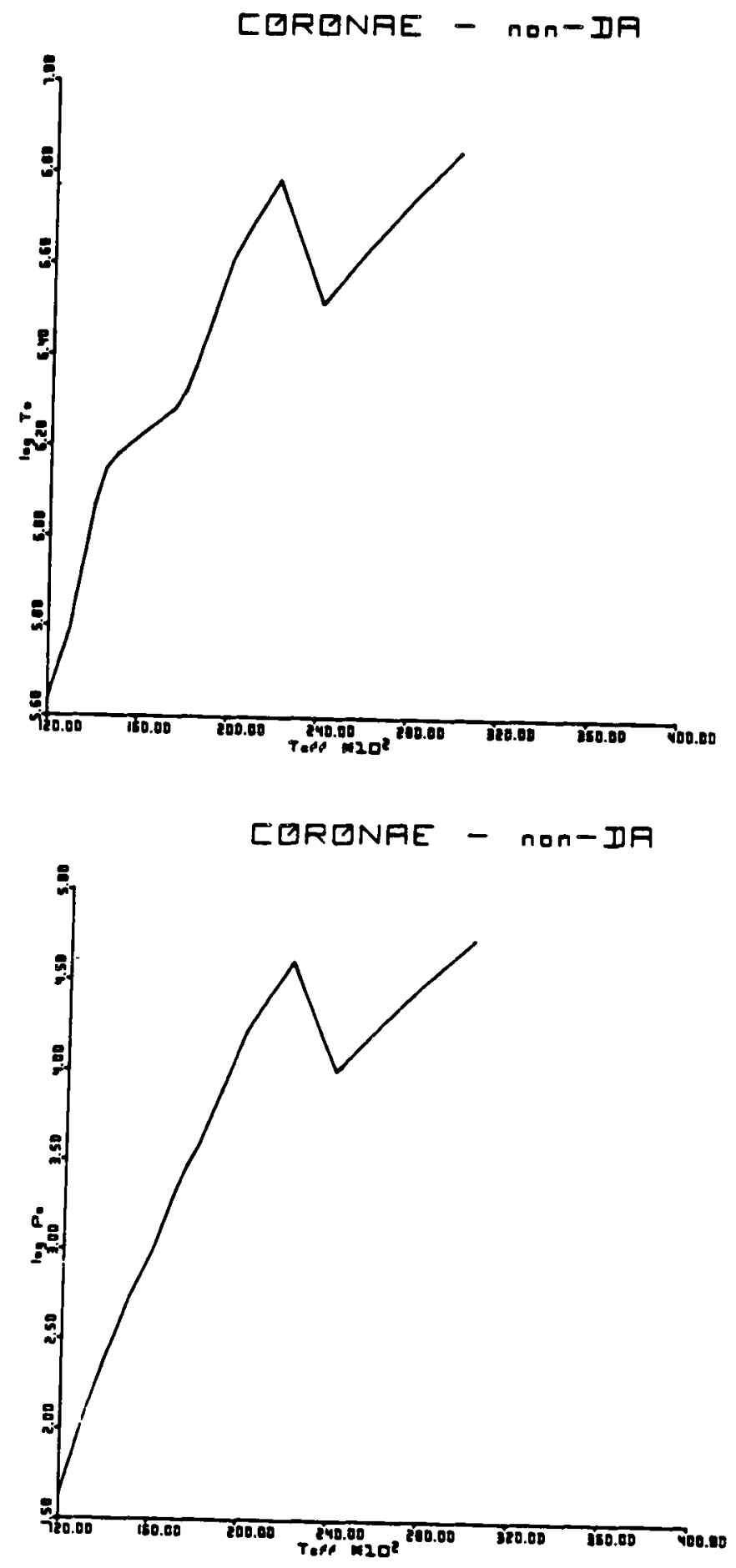

Fig. 9. Coronal temperature (upper part of diagram) and base pressure for non-DA models as a function of $T_{\text {eff }}$. 

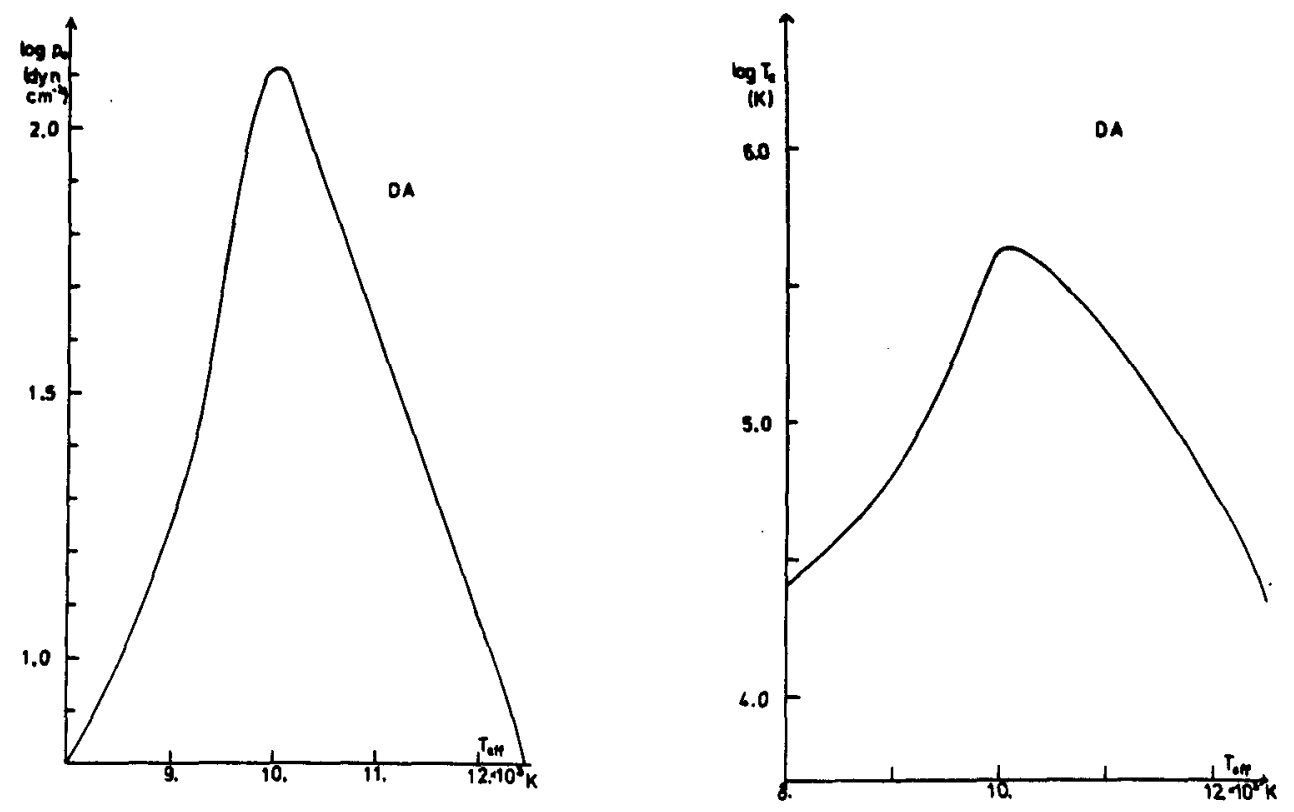

Fig. 10. The same as fig. 9 for DA models.

It should be noted that because of their very small scale height white dwarf coronae do not lead to a stellar wind.

This study has been supported by NSF grant AST 78-20542. 


\section{REFRENCES}

Antlochos, S.K. and Underwood, J.H. 1978, Astron. Astrophys., 68, L19.

d'Antona, F. and Mazzitelli, I. 1975, Astron. Astrophys., 44, 253.

d'Antona, F. and Mazzitel11, I. 1979, Astron. Astrophys., 74, 161.

Auer, L.H. and Mthalas, D. 1969, Ap.J., 158, 641.

Auer, L.H. and Mihalas, D. 1970, M.N.R.A.S., 149, 65.

Auer, L.H. and Shipman, H.L. 1977, Ap.J., 211, L103.

Biermann, L. and Lllst, R. 1960, in Stellar Atmospheres, Ed. J.I. Greenstein, University of Chicago Press, p. 260.

B Bhm, K.H. 1963, Ap.J., 138, 297。

B 8 hm, K.H. 1968, Astrophys. Space Sc1., 2, 375.

B bhm, K.H. 1969, Astron. Astrophys. 1, 180.

B $8 \mathrm{hm}$, K.H. 1970, Ap.J., 162, 919.

BUhm, K.H. 1976, Paper presented at the 2nd European Workshop on White Dwarfs, Monte Porzio, Italy.

B לhm, K.H. , Carson, T.R., Fontaine, G. and Van Horn, H.M. 1977, Ap.J., 217, 521 .

B $8 \mathrm{hm}$, K.H. and Cassinell1, J. 1971a, Astron. Astrophys., 12, 21.

B bhm, K.H. and Cassinell1, J, 1971b, in White Dwarfs, Proc. I.A.U. Symp. No. 42, ed. W.J. Luyten, Reidel Pub1. Co., p. 130.

B Hhm, K.H. and Deinzer, W. 1966, Z.f.A., 63, 177.

Bל̈hm, K.H. and Grenfe11, T.C. 1972, Astron. Astrophys. 20, 293.

$B B h m$, K.H. and Kapranidis, S. 1979, to be submitted to Astron. Astrophys.

Bues, I. 1970, Astron. Astrophys., 7, 91.

Bues, I. 1973, Astron. Astrophys., 28, 181.

Cash, W., Bowyer, S. and Lampton, M. 1978, Ap.J., 221, L87.

De Loore, C. 1970, Ap. and Space Sc1., 6, 60 .

Fontaine, G. 1973, Outer Layers of White Dwarf Stars, Ph.D. Thesis. Univ. of Rochester. 
Fontalne, G. and Van Horn, H.M. 1976, Ap.J. Suppl., 31, 476.

Fontaine, G., Van Horn, H.M., B bhm, K.H. and Grenfell, T.C. 1974, Ap.J., 193, 205.

Fontaine, G. and Michaud, G. 1979, Diffusion Time Scales in White Dwarfs, Preprint.

Greenstein, J.L. 1969, Comments Astrophys. Space Phys., 1, 62.

Grenfell, T.C. 1974, Astron. Astrophys., 40, 355.

Hearn, A.G. 1975, Astron. Astrophys. 40, 355.

Huppert, H.E. 1977, in Problems of Stellar Convection, ed. E.A. Splegel and J.P. Zahn, Springer-Verlag, Heldelberg-New York, p. 239.

Koester, D. 1972, Astron. Astrophys. 16, 459.

Koester, D. 1976; Astron. Astrophys., 52, 415.

Koester, D., Liebert, J. and Hege, E.K. 1979, Astron. Astrophys., 71, 163.

Koester, D., Schulz, H. and Weidemann, V. 1979, Astron. Astrophys. (In press).

Kuperus, M. 1965, The Transfer of Mechanical Energy in the Sun and the Heating of the Corona, Reidel Publ. Co., Dordrecht.

Liebert, J. 1977, Astron. Astrophys., 60, 101

Llebert, J., Dahn, C.C., Gresham, M. and Strittmatter, P.A. 1979, Preprint.

Lighth111, M.J. 1955, in Gas Dynamics of Cosmic Clouds, eds. J.M. Burgers and H.C. van der Hulst, Interscience Publ., New York, p. 429 .

Muchmore, D.O. and Bbhm, K.H. 1977, Astron. Astrophys., 54, 499. and $57,473$.

Muchmore, D.O. and Bbhm, K.H. 1978, Astron. Astrophys., 69, 113.

Mullan, D.J. 1976, Ap.J., 209, 171.

Schatzman, E. 1958, White Dwarfs, North-Holland Publ. Co., Amsterdam.

Shaviv, G. and Salpeter, E. 1973, Ap.J., 184, 191

Shipman, H.L. 1976, Ap.J., 206, L67.

Strittmatter, P.A. and Wickramasinghe, D.T. 1970, M.N.R.A.S., 150, 435. 
Strittmatter, P.A. and Wickramasinghe, D.T. 1971, M.N.R.A.S., 152, 47.

Terash1ta, Y., Matsushima, S. 1969, Ap.J., 156, 203.

Ulmschneider, P. 1967, Z. f. Ap., 67, 193.

Unno, W. 1963, P.A.S.J., 15, 400 .

Van Horn, H.M. 1970, Ap.J., 160, L53.

Vauclair, G. and Fontaine, G. 1979, Ap.J. (in press).

Wegner, G. 1972, Ap.J., 172, 451.

Wehrse, R. 1971, Astron. Astrophys., 19, 453.

Wehrse, R. 1974,, Astron. Astrophys., 39, 169.

Wehrse, R. 1976a, Mem. d. Soc. Astron. Itallana, 48, 13.

Wehrse, R. 1976b, Astron. Astrophys., 47, 482.

Wehrse, R. and Liebert, J. 1979, to be publ. In Astron. Astrophys.

Weidemann, V. 1960, Ap.J., 131, 638.

Weidemann, V. 1963, Z. f. Ap., 57, 87.

Weidemann, V. and Koester, D. 1979, Astron. Astrophys. (In press).

Wesemael, F. 1978, Astron. Astrophys., 65, 301.

Wesemael, F. 1979, in preparation. 